Comment. Math. Helv. 72 (1997) 618-635

(C) 1997 Birkhäuser Verlag, Basel

0010-2571/97/040618-18 \$1.50+0.20/0

Commentarii Mathematici Helvetici

\title{
A class of flows on 2-manifolds with simple recurrence
}

Konstantin Athanassopoulos, Theodoros Petrescou and Polychronis Strantzalos

\begin{abstract}
We study $D$-stable flows on orientable 2-manifolds of finite genus in connection with the topology of the underlying phase spaces. The description of the phase portrait is used to prove that a connected orientable 2-manifold of finite genus supporting a non-minimal $D$-stable flow must be homeomorphic to an open subset of the 2-sphere or the 2-torus. In the case of the presence of singularities we necessarily have an open subset of the 2-sphere.
\end{abstract}

Mathematics Subject Classification (1991). 58F25, 54H20.

Keywords. $D$-stable flow, 2-manifold of finite genus, recurrence, periodic orbit, local center.

\section{Introduction}

The main object of study in this article is the class of $D$-stable flows on 2-manifolds of finite genus (for definition see section 2). We are concerned with their qualitative behavior in connection with the topological structure of the underlying manifold. This point of view is in the center of the theory of transformation groups and dynamical systems. The class of $D$-stable flows is proved to be suitable for the main purposes of the two theories.

More precisely, we want to find the orientable 2-manifolds of finite genus that admit $D$-stable flows. To do this, we need to examine first the geometric properties of the orbits of $D$-stable flows, which is of independent interest in the theory of dynamical systems. The first, and possibly more important, step towards the qualitative analysis of a flow is to determine the topological and dynamical structure of its limit sets. The limit sets (and in fact all orbit closures) of a $D$-stable flow are minimal sets. A minimal set of a continuous flow on a manifold is a nonempty, closed, invariant set which has no proper subset with these properties and is called simple (or trivial) if it consists of a single orbit or is the whole manifold. A flow on a 2-manifold of finite genus has simple compact minimal sets if and only if it is smoothable, that is topologically equivalent to a $C^{\infty}$ flow [5]. This is a special property of flows on 2-manifolds, since there are $C^{\infty}$ flows even on $S^{3}$ with 1-dimensional non-simple minimal sets [8]. However the smoothability of a flow on a 2-manifold of finite genus (even compact) does not prevent the existence of 
non-periodic Poisson stable orbits.

In section 2 we consider the $\mathcal{C}$-flows, which are a larger class of flows containing the $D$-stable flows, and whose orbits are either closed sets or positively or negatively Poisson stable. We prove that a non-periodic positively (or negatively) Poisson stable orbit of a $\mathcal{C}$-flow on an orientable 2 -manifold of finite genus and with countably many ends is locally dense. Thus, any $\mathcal{C}$-flow on an orientable 2 manifold of finite genus is smoothable. The assumption on the countability of the ends can be removed in the subclass of $D$-stable flows (see Theorem 2.4). Having at hand a Poincaré-Bendixson type theorem for non-minimal $D$-stable flows on orientable 2-manifolds of finite genus, we give in section 3 a qualitative description of their phase portrait. For the minimal flows we refer the reader to [3]. This description is used in section 4 to find the orientable 2-manifolds of finite genus that admit non-minimal $D$-stable flows. In the main result of section 4 we prove that these manifolds are precisely the ones of genus zero or one, that is they are homeomorphic to the complement of a compact and totally disconnected set in the 2-sphere or the 2-torus. Moreover, an orientable 2-manifold of finite genus that admits a $D$-stable flow with at least one singularity has genus zero.

Some of the results of section 3 and Theorem 2.4(b) are contained in the second author's thesis [10], which was carried out under the guidance of the third author and is available only in Greek. We thank A. Manoussos for pointing out to us at an early stage that the method of proof of Theorem 2.4(b) also works to prove (a).

\section{Recurrence in $\mathcal{C}$-flows on orientable 2-manifolds of finite genus}

Let $\phi$ be a continuous flow on a manifold $M$. We shall denote by $\phi(t, x)=t x$ the translation of the point $x \in M$ along its orbit in time $t \in \mathbf{R}$. We shall also write $\phi(I \times A)=I A$, for $I \subset \mathbf{R}$ and $A \subset M$. The orbit of $x$ will be denoted by $C(x)$, its positive semiorbit by $C^{+}(x)$ and the negative by $C^{-}(x)$. The positive limit set of $x \in M$ is the closed invariant set

$$
L^{+}(x)=\left\{y \in M: t_{n} x \rightarrow y \text { for some } t_{n} \rightarrow+\infty\right\}
$$

and its (first) prolongational positive limit set is the set

$$
J^{+}(x)=\left\{y \in M: t_{n} x_{n} \rightarrow y \text { for some } x_{n} \rightarrow x \text { and } t_{n} \rightarrow+\infty\right\} .
$$

The negative versions $L^{-}(x)$ and $J^{-}(x)$ are defined by reversing time. A point $x \in M$ is non-wandering if $x \in J^{+}(x)$.

The orbit $C(x)$ is called positively (resp. negatively) Poisson stable if $x \in L^{+}(x)$ (resp. $x \in L^{-}(x)$ ), and Poisson stable if $x \in L^{+}(x) \cap L^{-}(x)$. A positively (or negatively) Poisson stable orbit $C(x)$ is called locally dense if $x \in \operatorname{int} \overline{C(x)}$. An exceptional orbit is a positively or negatively Poisson stable orbit which is nonsingular, non-periodic and non-locally-dense. 
Definition 2.1. (a) A $\mathcal{C}$-flow is a non-trivial continuous flow on a manifold each orbit of which is either a closed set or positively or negatively Poisson stable.

(b) A non-trivial flow $\phi$ on a manifold $M$ is called D-stable (or of characteristic 0 [7]) if $\overline{C(x)}=D(x)$ for every $x \in M$, where $D(x)$ is its (bilateral first) prolongation defined as follows,

$$
D(x)=\left\{y \in M: t_{n} x_{n} \rightarrow y, \text { for some } x_{n} \rightarrow x \text { and } t_{n} \in \mathbf{R}\right\} .
$$

Note that $D(x)=C(x) \cup J^{+}(x) \cup J^{-}(x)$ and that $y \in D(x)$ if and only if $x \in D(y)$. Thus, in a $D$-stable flow every orbit closure is a minimal set, and we have a $\mathcal{C}$-flow. Moreover, the reverse flow of a $D$-stable flow is also $D$-stable and the restricted flow in any connected open invariant set of a $D$-stable flow is $D$ stable. It is clear that the property of being $D$-stable is invariant under topological equivalences. Actually something more general is true.

Proposition 2.2. Let $M$ and $N$ be two manifolds carrying non-trivial continuous flows, and let $f: M \rightarrow N$ be a proper continuous map onto $N$ carrying orbits in $M$ onto orbits in $N$. If the flow on $M$ is D-stable, then the flow on $N$ is also D-stable.

Proof. Let $t_{n} \in \mathbf{R}, f\left(x_{n}\right) \rightarrow f(x)$ and $t_{n} f\left(x_{n}\right) \rightarrow f(y)$. Since $f$ is proper, the family $\mathcal{D}=\left\{f^{-1}(z): z \in N\right\}$ is an upper semicontinuous decomposition of $M$ into compact sets. This implies that there are $x^{\prime}, y^{\prime} \in M$ and $t_{n}^{\prime} \in \mathbf{R}, n \in \mathbf{N}$, such that $f(x)=f\left(x^{\prime}\right), f(y)=f\left(y^{\prime}\right)$ and $x_{n} \rightarrow x^{\prime}, t_{n}^{\prime} x_{n} \rightarrow y^{\prime}$. Hence $y^{\prime} \in D\left(x^{\prime}\right)=\overline{C\left(x^{\prime}\right)}$, because the flow on $M$ is $D$-stable. It follows that $f(y)=f\left(y^{\prime}\right) \in f\left(\overline{C\left(x^{\prime}\right)}\right)=$ $\overline{f\left(C\left(x^{\prime}\right)\right)}=\overline{C\left(f\left(x^{\prime}\right)\right)}=\overline{C(f(x))}$.

Starting with a minimal flow on the 2-torus one can easily construct examples of $\mathcal{C}$-flows on 2 -manifolds with locally dense orbits. Here we are interested in the recurrence properties of $\mathcal{C}$-flows on orientable 2-manifolds of finite genus.

An orientable 2-manifold of finite genus $n$ is constructed as follows. Let $F$ be a closed and totally disconnected subset (maybe empty) of the 2-sphere $S^{2}$. From $S^{2} \backslash F$ we remove the interiors of $2 n$ pairwise disjoint closed discs and identify their boundaries to form $n$ handles $h_{1}, \ldots, h_{n}$. Then $M$ is homeomorphic to $S^{2} \cup h_{1} \cup \ldots \cup h_{n} \backslash F$. Adding $F$ to $M$ we obtain a compact 2-manifold $M^{+}$of genus $n$, which is the end point compactification of $M$. The elements of $M^{+} \backslash M$ are called ends of $M$. It can be proved that a flow on $M$ can be extended to a flow on $M^{+}$that fixes the ends pointwise [1, Satz 2.3].

Let $\phi$ be a $\mathcal{C}$-flow on an orientable 2-manifold of finite genus $M$ and denote by $R$ the set of points in $M$ whose orbit is a non-compact closed set. The orbit of a point $x \in R$ tends in positive time to an end, which we denote by $e^{+}(x)$. So we get a well defined map $e^{+}: R \rightarrow M^{+} \backslash M$ and similarly a map $e^{-}$, by considering negative time. 
Proposition 2.3. If $\phi$ is a D-stable flow on an orientable 2-manifold of finite genus $M$, then the maps $e^{+}, e^{-}$are continuous.

Proof. Let $\left\{x_{n}: n \in \mathbf{N}\right\}$ be a sequence of points in $R$ converging to a point $x \in R$ and suppose, by contradiction, that the sequence $\left\{e^{+}\left(x_{n}\right): n \in \mathbf{N}\right\}$ does not converge to $e^{+}(x)$. Since $M^{+} \backslash M$ is compact, passing to a subsequence if necessary, we may assume that there is an end $e \neq e^{+}(x)$ such that $e^{+}\left(x_{n}\right) \rightarrow e$. There is a compact neighbourhood $V$ of $e$ in $M^{+}$such that $e^{+}(x) \notin V$ and $\partial V \subset M$, because $M^{+} \backslash M$ is totally disconnected. Since $\lim _{t \rightarrow+\infty} t x=e^{+}(x)$, the continuity of the extended flow on $M^{+}$and the connectedness of the orbits imply that there are $t_{n} \rightarrow+\infty$ such that $t_{n} x_{n} \in \partial V$ and $C^{+}\left(t_{n} x_{n}\right) \subset V$ eventually for all $n \in \mathbf{N}$. The sequence $\left\{t_{n} x_{n}: n \in \mathbf{N}\right\}$ has a limit point $y \in \partial V$ and $C^{+}(y) \subset V$. Hence $y \notin C(x)$. On the other hand, $y \in D(x)=C(x)$. This contradiction shows that the map $e^{+}$is continuous.

The above Proposition is true for $D$-stable flows on any manifold of arbitrary dimension. For the general definition of the end point compactification we refer the reader to [1].

Theorem 2.4. Let $\phi$ be a $\mathcal{C}$-flow on an orientable 2-manifold of finite genus $M$. If

(a) $M$ has countably many ends, or

(b) $\phi$ is D-stable,

then $\phi$ has no exceptional orbit.

Proof. Suppose, by contradiction, that there is an exceptional orbit $C(x)$. There exists a local section $S$ at $x$ which is an arc [6, Ch. VII, Theorem 1.6]. The set $S \cap \overline{C(x)}$ is a totally disconnected perfect set, because $C(x)$ is exceptional. Thus, there is a sequence of mutually disjoint intervals in $S \backslash \overline{C(x)}$ accumulating to $x$. Since $M$ has finite genus, there are finitely many pairwise disjoint non-periodic positively (or negatively) Poisson stable orbit closures [5], [9]. Moreover, according to the Structure Theorem in [5], if $x \in \overline{C(y)}$ and $y$ is positively (or negatively) Poisson stable, then $\overline{C(x)}=\overline{C(y)}$. On the other hand, $x$ cannot be the limit of a sequence of singular or periodic points [9, Lemma 6]. It follows from these and our assumption on the flow that shrinking $S$ we may assume that $S \backslash \overline{C(x)} \subset \operatorname{int} R$. Each point of $S \backslash \overline{C(x)}$ returns to $S$ at most finitely many times. If we assume (a), then every connected component of $S \backslash \overline{C(x)}$ contains at least two points (in fact uncountably many) whose orbits tend in positive (resp. negative) time to the same end. If we assume (b), then the orbits of all points in a connected component of $S \backslash \overline{C(x)}$ tend in positive (resp. negative) time to the same end, because $M^{+} \backslash M$ is totally disconnected and the maps $e^{+}, e^{-}$are continuous, by Proposition 2.3. Thus, in either case there exists a sequence $\left\{x_{n}: n \in \mathbf{N}\right\}$ of points of $R$ converging to $x$, with mutually disjoint orbits, and two sequences of ends $\left\{e_{n}^{+}: n \in \mathbf{N}\right\}$ and 
$\left\{e_{n}^{-}: n \in \mathbf{N}\right\}$ such that

$$
\lim _{t \rightarrow \pm \infty} t x_{2 n-1}=\lim _{t \rightarrow \pm \infty} t x_{2 n}=e_{n}^{ \pm}
$$

for every $n \in \mathbf{N}$. Passing to suitable subsequences if necessary, it suffices to consider only the following two cases.

Case I. Suppose that $e_{n}^{+}=e_{n}^{-}$for every $n \in \mathbf{N}$. The sets $C_{2 n-1}=C\left(x_{2 n-1}\right) \cup$ $\left\{e_{n}^{+}\right\}$and $C_{2 n}=C\left(x_{2 n}\right) \cup\left\{e_{n}^{+}\right\}$are simple closed curves for all $n \in \mathbf{N}$. If $C_{n}$ separates $M^{+}$, then it is the boundary of an invariant open set in $M^{+}$. By the continuity of the flow and because $C(x)$ is exceptional, the orbit $C\left(x_{n}\right)$ crosses $S$ at least three times, provided $n$ is large enough, and we arrive at a contradiction as in the proof of Lemma 6 in [9]. This shows that there exists $N \in \mathbf{N}$ such that $C_{n}$ does not separate $M^{+}$for $n \geq N$.

Case II. Suppose that $e_{n}^{+} \neq e_{n}^{-}$for every $n \in \mathbf{N}$. The set $C_{n}=C\left(x_{2 n-1}\right) \cup$ $C\left(x_{2 n}\right) \cup\left\{e_{n}^{+}, e_{n}^{-}\right\}$is a simple closed curve for all $n \in \mathbf{N}$. As in case I there exists $N \in \mathbf{N}$ such that $C_{n}$ does not separate $M^{+}$for $n \geq N$.

In both cases, the set $M_{1}=M \cap\left(M^{+} \backslash C_{N}\right)$ is a connected, open, invariant subset of $M$, which as a 2-manifold has genus smaller than $M$ and has countably many ends, if $M$ has. The restricted flow in $M_{1}$ is a $\mathcal{C}$-flow, which again has the exceptional orbit $C(x)$. Repeating now the above process, we arrive after a finite number of steps at a connected, open, invariant subset of $M$ of genus zero which contains $C(x)$. This contradicts the Poincaré-Bendixson theorem and proves the conclusion under the assumption (a). If we assume (b), then the restricted flow on $M_{1}$ is a $D$-stable flow on an orientable 2-manifold of genus smaller than $M$ having the exceptional orbit $C(x)$. Again by induction we arrive at a contradiction to the Poincaré-Bendixson theorem.

Proposition 2.5. Let $\phi$ be a $\mathcal{C}$-flow with totally disconnected set of singularities on an orientable 2-manifold of finite genus $M$. If there exists a locally dense orbit $C(x)$ with compact closure, then $M=\overline{C(x)}$.

Proof. Since $\overline{C(x)}$ is compact and $\phi$ is a $\mathcal{C}$-flow, every orbit in $\overline{C(x)}$ is either singular or positively or negatively Poisson stable, because $\overline{C(x)}$ cannot contain periodic points by the trapping argument. By the Structure Theorem in [5], $\partial \overline{C(x)}$ consists of singularities and is therefore totally disconnected by assumption. Hence $M=\overline{C(x)}$.

For any $n \in \mathbf{N}$ there are $\mathcal{C}$-flows without singularities on orientable 2-manifolds of genus $n$ and with finitely many ends, having exactly $n$ pairwise disjoint locally dense, non-compact, orbit closures. For example, there is a flow on the 2-torus $T^{2}$ having an invariant closed disc $D$ such that the flow in $D$ looks like that of figure 1 and the restricted flow in $T^{2} \backslash D$ is minimal. Take two orbits $C_{1}, C_{2} \operatorname{in} \operatorname{int} D$ and remove the interior of the disc in $T^{2}$ bounded by $\overline{C_{1} \cup C_{2}}$. Take two copies of 
the remainder with reversely oriented flows and identify their boundaries pointwise to get an orientable compact 2-manifold of genus 2 carrying a flow with two (hyperbolic) singularities and two locally dense orbit closures, whose intersection is the two singularities. Removing the singularities, we obtain a $\mathcal{C}$-flow without singularities on an orientable 2-manifold of genus 2 with two ends and two disjoint locally dense, non-compact, orbit closures.
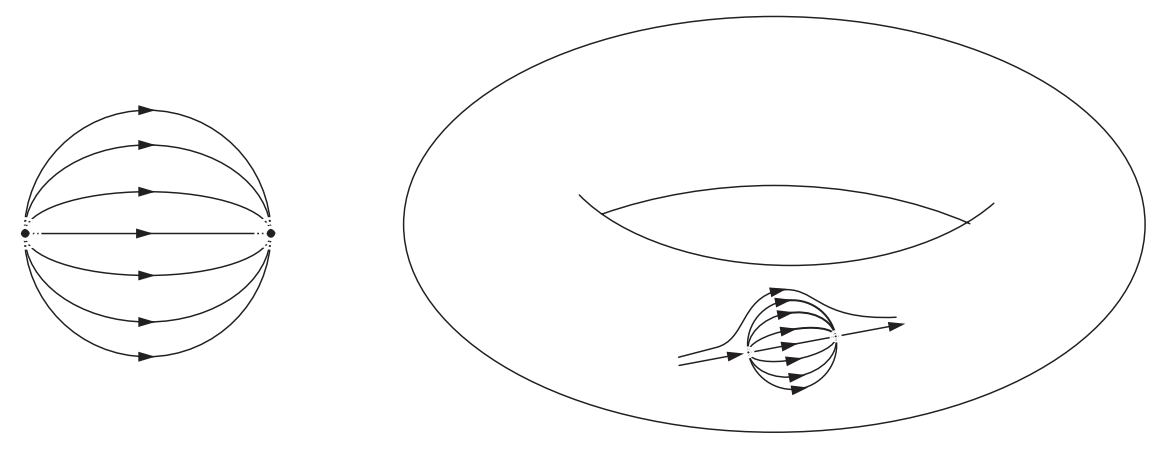

Figure 1.

\section{3. $D$-stable flows on orientable 2-manifolds of finite genus}

In this section we shall analyze the global qualitative behavior of $D$-stable flows defined on orientable 2-manifolds of finite genus. So from now on $\phi$ will be a $D$ stable flow on an orientable 2-manifold of finite genus. In order to study the global qualitative behavior of $\phi$, we must first describe its recurrence properties. Note that $\phi$ has a locally dense orbit if and only if it is a minimal flow. So, Theorem 2.4 has the following consequence.

Corollary 3.1. Let $\phi$ be a non-minimal D-stable flow on an orientable 2-manifold of finite genus $M$. If $x \in M$ is a positively (or negatively) Poisson stable point, then $x$ is singular or periodic.

For the rest of the section we assume further that $\phi$ is a non-minimal $D$-stable flow. Let $R$ denote the set of points in $M$ whose orbit is a non-compact closed set and let $e^{+}, e^{-}: R \rightarrow M^{+} \backslash M$ be the continuous maps defined just before Proposition 2.3. If $F$ denotes the set of singularities and $P$ the set of periodic points, then $M=F \cup P \cup R$.

Lemma 3.2. The set $F \cup P$ is open in $M$.

Proof. If $x \in F \cup P$, then its orbit $C(x)$ is a bilaterally Lyapunov stable compact 
invariant set $[4, \mathrm{Ch} . \mathrm{V}$, Theorem 1.12]. Hence $C(x)$ has a compact invariant neighbourhood $V$. It is clear now that $V \subset F \cup P$.

Lemma 3.3. If $R \neq M$ and $C$ is a connected component of $R$, then $e^{+}\left|C=e^{-}\right| C$ and is constant. Moreover, the closure of $C$ in $M^{+}$is $C \cup\left\{e^{+}(C)\right\}$.

Proof. By proposition 3.1, the maps $e^{+}, e^{-}$are continuous and so take constant values on $C$. Let $e_{1}=e^{-}(C)$ and $e_{2}=e^{+}(C)$. If $x \in \partial C$, which exists because $R \neq M$, there are periodic points $x_{n} \rightarrow x$. Suppose that $e_{1} \neq e_{2}$ and let $V$ be a compact neighbourhood of $e_{2}$ in $M^{+}$such that $e_{1} \notin V$ and $\partial V \subset M$. By the continuity of the flow and the connectedness of orbits, there are $t_{n} \rightarrow+\infty$ and $T>0$ such that $C^{+}(T x) \subset V$ and $\left[T, t_{n}\right] x_{n} \subset V$ and $t_{n} x_{n} \in \partial V$. The sequence $\left\{t_{n} x_{n}: n \in \mathbf{N}\right\}$ has a limit point $y \in \partial V$ such that $C^{-}(y) \subset V$. However, $y \in J^{+}(x) \subset C(x)$, and we have a contradiction, since $\lim _{t \rightarrow-\infty} t x=e_{1}$. This proves the first assertion.

To prove the second assertion, suppose that the end $e$ belongs to the closure of $C$ in $M^{+}$and $e \neq e^{+}(C)$. Let $W$ be a compact neighbourhood of $e$ such that $\partial W \subset M$ and $e^{+}(C) \notin W$. There exists a sequence $\left\{y_{n}: n \in \mathbf{N}\right\}$ of points in $C \cap W$ converging to $e$. Since $e^{+}\left(y_{n}\right)=e^{-}\left(y_{n}\right)=e^{+}(C)$, there are $s_{n}<0<t_{n}$ such that $\left[s_{n}, t_{n}\right] y_{n} \subset W$ and $s_{n} y_{n}, t_{n} y_{n} \in \partial W$ for every $n \in \mathbf{N}$. Moreover, $t_{n} \rightarrow+\infty$ and $s_{n} \rightarrow-\infty$. The sequence $\left\{t_{n} y_{n}: n \in \mathbf{N}\right\}$ has a limit point in $z \in \partial W$ such that $C^{-}(z) \subset W$. Hence $e^{+}(C)=e^{-}(z) \in W$, contradiction.

Theorem 3.4. If $P=\emptyset$, then the flow is parallelizable and $M$ is homeomorphic either to $\mathbf{R}^{2}$ or to $\mathbf{R} \times S^{1}$.

Proof. From Lemma 3.2 and connectedness we have $M=R$ and Proposition 2.3 implies that there are $e_{1}, e_{2} \in M^{+} \backslash M$ such that $e^{-}(x)=e_{1}$ and $e^{+}(x)=e_{2}$ for every $x \in M$. Suppose, by contradiction, that the flow is not parallelizable. Then, there exists some point $x \in M$ such that $J^{+}(x) \neq \emptyset[4$, Ch. IV, Theorem 2.6] and hence $x \in J^{+}(x)$, since $J^{+}(x) \subset D(x)=C(x)$. Thus, there are $x_{n} \rightarrow x$ and $t_{n} \rightarrow+\infty$ such that $t_{n} x_{n} \rightarrow x$. If $e_{1} \neq e_{2}$, then an argument similar to that used in the proof of Lemma 3.3 leads to a contradiction. So, necessarily $e_{1}=e_{2}$ and the set $C=C(x) \cup\left\{e_{1}\right\}$ is a simple closed curve in $M^{+}$. Since $M^{+}$is orientable, there exists an open neighbourhood $V$ of $C$ in $M^{+}$such that $\emptyset \neq \partial V \subset M$, that is homeomorphic to $(-1,1) \times S^{1}$ so that $C$ corresponds to $\{0\} \times S^{1}$. The orbits $C\left(x_{n}\right), n \in \mathbf{N}$, lie eventually in $V$, since $D(x)=C(x)$. Let $S$ be a local section at $x$, which is an arc and intersects $C(x)$ only at $x$. We may assume that $x_{n}$, $t_{n} x_{n} \in S$ and that $x_{n}$ is the first point $C\left(x_{n}\right)$ crosses $S$. If $V_{1}$ and $V_{2}$ are the two connected components of $V \backslash C$, then we may further assume that $C\left(x_{n}\right) \subset V_{1}$ for all $n \in \mathbf{N}$. The set $S_{n}=\left[0, t_{n}\right] x_{n} \cup\left[x_{n}, t_{n} x_{n}\right]$ is a simple closed curve in $V$, 
where $\left[x_{n}, t_{n} x_{n}\right]$ denotes the segment on $S$ with endpoints $x_{n}$ and $t_{n} x_{n}$. If $S_{n}$ is nullhomotopic in $V$, then it bounds a positively (resp. negatively) invariant open disc $D \subset V$. Otherwise, $S_{n}$ and $C$ bound a positively (resp. negatively) invariant subannulus in $V_{1}$. But then in both cases it is impossible to have $e^{+}\left(x_{n}\right)=e^{-}\left(x_{n}\right)$. This proves that the flow must be parallelizable. The rest is a consequence of $[6$, Ch. VII, Theorem 1.6].

Proposition 3.5. The non-wandering set is $\overline{F \cup P}$ and the restricted flow on $R$ is parallelizable.

Proof. If $R=M$, then the flow is parallelizable, by Theorem 3.4, and there is nothing to prove. Suppose that $R \neq M$. Then, $e^{+}(x)=e^{-}(x)$ for every $x \in R$, by Lemma 3.3. Let $y \in R$ and assume that there are points $x_{n} \in R, n \in \mathbf{N}$, and times $t_{n} \rightarrow+\infty$ such that $x_{n} \rightarrow x$ and $t_{n} x_{n} \rightarrow y$. Then, we arrive at a contradiction in the same way as in the proof of Theorem 3.4. This proves both assertions simultaneously.

Corollary 3.6. Let $x, x_{n} \in R$ and $t_{n} \in \mathbf{R}, n \in \mathbf{N}$, be such that $t_{n} \rightarrow \pm \infty$ and $x_{n} \rightarrow x$. Then $t_{n} x_{n} \rightarrow e^{ \pm}(x)$.

Proof. If $R=M$, the flow is parallelizable, by Theorem 3.4, and the assertion is evident. Let $R \neq M$. Then, $e^{+}(x)=e^{-}(x)$, from Lemma 3.3, and Proposition 3.5 implies that there is some $e \in M^{+} \backslash M$ such that $t_{n} x_{n} \rightarrow e$. Suppose by contradiction that $e \neq e^{+}(x)$. There is then a compact neighbourhood $V$ of $e$ in $M^{+}$such that $\partial V \subset M$ and $V \cap\left(C(x) \cup\left\{e^{+}(x)\right\}\right)=\emptyset$. Eventually, $x_{n} \in M^{+} \backslash V$ and $t_{n} x_{n} \in V$. Hence there are $s_{n}>0, n \in \mathbf{N}$, such that $s_{n} x_{n} \in \partial V$. The sequence $\left\{s_{n} x_{n}: n \in \mathbf{N}\right\}$ has a limit point $y \in \partial V$. Obviously, $y \in D(x)=C(x)$, contradiction.

Our job for the rest of this section will be to study the topological structure of each one of the sets $F, P$ and $R$. We start with the structure of $F$.

Lemma 3.7. The set $F$ consists of local centers.

Proof. Let $z \in \partial F$. By Lemma 3.2, there exists an invariant, open, connected neighbourhood $V$ of $z$ in $M$ such that $V \subset F \cup P$. Then, $J^{+}(x)=C(x)=L^{+}(x)$ for every $x \in V$. It follows from [2, Theorem 3.1.4] that $z$ is a local center. In particular, $D \cap F=\{z\}$ for some invariant disc $D \subset V$, and $\{z\}$ is open and closed in $F$. It follows that $F$ consists of local centers.

We shall prove later in section 4 that there can be at most two singularities 
(see Theorem 4.4).

Let now $P \neq \emptyset$ and suppose that $K$ is a connected component of $P$. By Lemma 3.2 and [13], the restricted flow on $K$ is topologically equivalent to the standard periodic flow on the 2-torus $T^{2}$ or on $\mathbf{R} \times S^{1}$. In the former case, $M=K \cong T^{2}$, by invariance of domain. So we deal only with the later. In this case there is a homeomorphism $h: \mathbf{R} \times S^{1} \rightarrow K$ such that the periodic orbits in $K$ are the sets $h\left(\{t\} \times S^{1}\right), t \in \mathbf{R}$. Since $K$ is open, elementary topology shows that the boundary $\partial^{+} K$ of $K$ in $M^{+}$is $K_{1} \cup K_{2}$ where

$$
K_{1}=\bigcap_{t<0} \overline{h\left((-\infty, t] \times S^{1}\right)} \text { and } K_{2}=\bigcap_{t>0} \overline{h\left([t,+\infty) \times S^{1}\right)}
$$

(closures taken in $\mathrm{M}^{+}$). The sets $K_{1}, K_{2}$ are invariant, compact and connected. If $K_{1} \cap K_{2} \neq \emptyset$, then $\partial^{+} K$ is connected. Otherwise, they are its connected components.

Proposition 3.8. Let $P \neq \emptyset$. If $K$ is a connected component of $P$, then its boundary $\partial K$ in $M$ has at most two connected components and each of them is an orbit in $F \cup R$.

Proof. Using the above notation, we have $\partial K \subset\left(K_{1} \cap M\right) \cup\left(K_{2} \cap M\right)$. If $x \in$ $K_{2} \cap M$, and $x \in R$, then the set $C=C(x) \cup\left\{e^{+}(x)\right\}$ is a simple closed curve, by Lemma 3.3. Since $M^{+}$is orientable, $C$ has a neighbourhood basis consisting of annular tubular neighbourhoods not intersecting $h\left(\{0\} \times S^{1}\right)$. Let $V$ be such an annulus. Since $D(x)=C(x)$, there is an orbit $\Gamma=h\left(\{t\} \times S^{1}\right) \subset V \cap K$, for some $t>0$. Then $\Gamma$ cannot bound a disc in $V$, because if it did, then $K_{2}$ would be contained in the interior of that disc, but not $C$, which is a contradiction. Thus, $\Gamma$ and $C$ bound an invariant subannulus and this with connectedness imply that $\overline{h\left([t,+\infty) \times S^{1}\right)} \subset V$. It follows that $K_{2} \subset V$ for every annular tubular neighbourhood $V$ of $C$. This shows that $K_{2}=C(x) \cup\left\{e^{+}(x)\right\}$. If $x \in F$, then $x$ is a local center by Lemma 3.7 and has a neighbourhood basis consisting of closed invariant discs. For any such disc $D$ there is some $t>0$ such that that $\overline{h\left([t,+\infty) \times S^{1}\right)} \subset D$. It follows that $K_{2} \subset D$, which shows that $K_{2}=\{x\}$. Similarly for $K_{1}$.

Our next task is to analyze the structure of the flow in $R$, if $R \neq M$, which we assume for the rest of this section.

Lemma 3.9. Let $C$ be a connected component of $R$. If $x \in \partial C$, there exists a sequence of periodic points $\left\{x_{n}: n \in \mathbf{N}\right\}$ converging to $x$ such that $C\left(x_{n}\right)$ and $C(x) \cup\left\{e^{+}(C)\right\}$ bound an invariant closed annulus $W_{n}$ in $M^{+}$such that the sequence $\left\{W_{n}: n \in \mathbf{N}\right\}$ decreases and

$$
\bigcap_{n=1}^{\infty} W_{n}=C(x) \cup\left\{e^{+}(C)\right\} .
$$


Proof. Since $R \neq M$, the set $B=C(x) \cup\left\{e^{+}(C)\right\}$ is a simple closed curve in $M^{+}$. Let $V$ be an annular tubular neighbourhood of $B$ in $M^{+}$, which exists because $M^{+}$is assumed to be orientable. Let $S \subset V$ be a local section to the flow at $x$ which is an arc and such that $S \cap C(x)=\{x\}$. The set $S \backslash\{x\}$ consists of two segments $S_{1}$ and $S_{2}$. Then, $S_{1} \subset V_{1}$ and $S_{2} \subset V_{2}$, where $V_{1}$ and $V_{2}$ are the two subannuli in which $B$ separates $V$. Since $x \in \partial C$, there is a sequence of periodic points on $S$ converging monotonically to $x$ from at least one side. Let " $<$ denote an orientation of $S$ and let $\left\{x_{n}: n \in \mathbf{N}\right\}$ be a sequence of periodic points on $S_{1}$ which decreases to $x$. Since $D(x)=C(x)$, we may assume that $C\left(x_{n}\right) \subset V_{1}$ for every $n \in \mathbf{N}$. Then, $C\left(x_{n}\right)$ crosses $S$ only at $x_{n}$. Passing to a subsequence if necessary, it suffices to deal only with the following two cases.

Case I. Suppose that $C\left(x_{n}\right)$ bounds an open, invariant disc $D_{n} \subset V_{1}$ for every $n \in \mathbf{N}$. Let $\left[x, x_{n}\right]$ denote the closed segment on $S$ with endpoints $x$ and $x_{n}$. Since $x \notin D_{n}$, if $\left[x, x_{n}\right] \cap D_{n} \neq \emptyset$, then some point between $x$ and $x_{n}$ on $S$ would be on $\partial D_{n}=C\left(x_{n}\right)$, contrary to $S \cap C\left(x_{n}\right)=\left\{x_{n}\right\}$. Hence $\left[x, x_{n}\right] \cap D_{n}=\emptyset$ and therefore $S \cap D_{n}=S_{1} \backslash\left[x, x_{n}\right]$. For every $n \in \mathbf{N}$ we have $S_{1} \backslash\left[x, x_{n}\right] \subset D_{n} \cap D_{n+1}$, because $x<x_{n+1}<x_{n}$ on $S$, and $\partial D_{n} \cap \partial D_{n+1}=\emptyset$. Consequently, $D_{n} \subset D_{n+1}$, because $\left(x_{n+1}, x_{n}\right] \subset D_{n+1} \backslash D_{n}$, by the Jordan Curve Theorem. The set $D=\cup_{k=1}^{\infty} D_{k}$ is an invariant open disc in $V_{1}$, by a theorem of M. Brown. The boundary $\partial^{+} D$ of $D$ in $M^{+}$is

$$
\partial^{+} D=\bigcap_{k=1}^{\infty} \overline{\bigcup_{n=k}^{\infty} D_{n}}
$$

(closures taken in $M^{+}$) and of course $B \subset \partial^{+} D$. We shall show that we actually have equality. Suppose that there is a point $z \in \partial^{+} D \backslash B$. There is a neighbourhood $W$ of $z$ in $M^{+}$, which is a closed disc such that $W \cap B=\emptyset$ and $\partial W \subset M$. Then, $W \cap D_{n_{k}} \neq \emptyset$ for some $n_{k} \rightarrow+\infty$. Since $z \in W \backslash D$, we have $W \cap \partial D_{n_{k}} \neq \emptyset$, for every $k \in \mathbf{N}$, by connectedness of $W$. On the other hand we have eventually $x_{n_{k}} \in M^{+} \backslash W$. Hence $\partial W \cap \partial D_{n_{k}} \neq \emptyset$, by connectedness of $\partial D_{n_{k}}=C\left(x_{n_{k}}\right)$. This means that there are $t_{n_{k}} \in \mathbf{R}$ such that $t_{n_{k}} x_{n_{k}} \in \partial W$ for every $k \geq k_{0}$, some $k_{0} \in \mathbf{N}$. The sequence $\left\{t_{n_{k}} x_{n_{k}}: k \geq k_{0}\right\}$ has a limit point $y \in \partial W$. But $y \in D(x)=C(x)$, which contradicts our choice of $W$. This shows that we must necassarily have $B=\partial^{+} D$. That is $B$ bounds a disc in $V$. This contradiction to the choice of $V$ proves that case I cannot occur.

Case II. Every periodic orbit $C\left(x_{n}\right)$ bounds with $B$ an invariant, open annulus $A_{n} \subset V_{1}$. Then the sets $W_{n}=\overline{A_{n}}, n \in \mathbf{N}$, are invariant closed annulli and constitute a neighbourhood basis of $B$ in $\overline{V_{1}}$, because $D(x)=C(x)$.

Corollary 3.10. Let $C$ be a connected component of $R$. If $\operatorname{int} C=\emptyset$, then $C$ consists of a single orbit.

Proof. Let $x \in C$. The set $B=C(x) \cup\left\{e^{+}(x)\right\}$ is a simple closed curve in $M^{+}$. Let $V$ be an annular tubular neighbourhood of $B$ in $M^{+}$and $V_{1}, V_{2}$ the subannuli 
in which $B$ divides $V$. Since $\operatorname{int} C=\emptyset$, the point $x$ is approximated by periodic points from both sides of $B$. So, by the proof of Lemma 3.9, there are periodic orbits $C\left(x_{n}\right) \subset V_{1}, n \in \mathbf{N}$, such that each $C\left(x_{n}\right)$ bounds with $B$ a closed annulus $W_{n}$ and $\cap_{n=1}^{\infty} W_{n}=B$. Similarly for $V_{2}$. It follows from this that $C \cap V=C(x)$. Hence $C=C(x)$.

Proposition 3.11. Let $C$ be a connected component of $R$. If $\operatorname{int} C \neq \emptyset$, then $\operatorname{int} C$ is homeomorphic to $\mathbf{R}^{2}$ and $\partial C$ consists of at most two orbits. If $\partial C$ contains only one orbit, then the restricted flow in $C$ has a section homeomorphic to the interval $[0,1)$ and $C$ is homeomorphic to $\mathbf{R} \times[0,1)$. If $\partial C$ consists of two orbits, then the section in $C$ is homeomorphic to $[0,1]$ and $C$ is homeomorphic to $\mathbf{R} \times[0,1]$.

Proof. Let $A$ be a connected component of int $C$. Let $\Sigma$ be section to the restricted flow in $C$ and $S$ a section to the flow in $A$. There is a continuous function $\tau$ : $C \rightarrow \mathbf{R}$ such that $\tau(x) x \in \Sigma$ for every $x \in C$ [4, Ch. IV, Theorem 2.4]. Let $f: S \rightarrow \Sigma$ be the continuous map defined by $f(x)=\tau(x) x$. It is easy to see that $f$ is a topological embedding and that $f(S)$ is open in $\Sigma$. Since $A$ is connected and open in $M$, the section $S$ is homeomorphic to $\mathbf{R}$ or to $S^{1}$ [6,Ch. VII, Theorem 1.6]. In the later case $f(S)$ would be an open and compact subset of $\Sigma$, which is connected, and therefore $C=A$ would itself be an open and closed subset of $M$. Hence the flow on $M$ would be parallelizable and $R=M$ would be homeomorphic to $\mathbf{R} \times S^{1}$. Thus, there is a homeomorphism $h: \mathbf{R} \rightarrow S$ and the map $\psi: \mathbf{R}^{2} \rightarrow A$ defined by $\psi(t, s)=t h(s)$ is a homeomorphism also. The boundary $\partial^{+} A$ of $A$ in $M^{+}$is

$$
\partial^{+} A=\bigcap_{t>0} \overline{\psi\left(\mathbf{R}^{2} \backslash[-t, t]^{2}\right)}
$$

(closure taken in $\left.M^{+}\right)$. Let $x \in \cap_{t>0} \overline{\psi((t,+\infty) \times(-t, t))}$ and suppose that $x \neq$ $e^{+}(C)$. The second assertion in Lemma 3.3 implies that there are $t_{n} \rightarrow+\infty$ and $s_{n} \in \mathbf{R}, n \in \mathbf{N}$, such that $t_{n} h\left(s_{n}\right) \rightarrow x$. The sequence $\left\{s_{n}: n \in \mathbf{N}\right\}$ must diverge, because otherwise there is a subsequence $\left\{s_{n_{k}}: k \in \mathbf{N}\right\}$ converging to some $s \in \mathbf{R}$. But then we would have $x, h\left(s_{n_{k}}\right), h(s) \in C, h\left(s_{n_{k}}\right) \rightarrow h(s)$ and $\psi\left(t_{n_{k}}, s_{n_{k}}\right) \rightarrow x$, which contradicts Proposition 3.5. Thus necessarily $s_{n} \rightarrow \pm \infty$. This and similar considerations for $\cap_{t>0} \overline{\psi((-\infty, t) \times(-t, t))}$ show that $\partial^{+} A=A_{1} \cup A_{2}$ where

$$
A_{1}=\bigcap_{s<0} \overline{\psi(\mathbf{R} \times(-\infty, s])} \text { and } A_{2}=\bigcap_{s>0} \overline{\psi(\mathbf{R} \times[s,+\infty))}
$$

(closures in $M^{+}$). Of course $e^{+}(C) \in A_{1} \cap A_{2}$.

We shall prove now that each of $A_{1}, A_{2}$ contains at most one orbit in $M$. Let $x \in A_{2} \cap M$. The set $B=C(x) \cup\left\{e^{+}(C)\right\}$ is a simple closed curve and has a neighbourhood basis in $M^{+}$consisting of closed annular tubular neighbourhoods, because $M^{+}$is orientable. Let $V$ be such a neighbourhood of $B$. There exist 
$t_{n} \in \mathbf{R}, n \in \mathbf{N}$, and $s_{n} \rightarrow+\infty$ monotonically, such that $\psi\left(t_{n}, s_{n}\right) \rightarrow x$. Since $D(x)=C(x)$, we may assume that $C\left(h\left(s_{n}\right)\right) \subset \operatorname{int} V_{1}$ for every $n \in \mathbf{N}$, where $V_{1}$ is one of the two subannuli in which $B$ divides $V$. The (non-simple) closed curve $C\left(h\left(s_{n}\right)\right) \cup C\left(h\left(s_{n+1}\right)\right) \cup e^{+}(C)$ is the complete boundary in $M^{+}$of the invariant open disc $D_{n}=\psi\left(\mathbf{R} \times\left(s_{n}, s_{n+1}\right)\right), n \in \mathbf{N}$. Clearly, $D_{n} \cap D_{m}=\emptyset$ for $n \neq m$ and eventually $D_{n} \subset \operatorname{int} V_{1}$. Indeed, if $D_{N} \not \subset \operatorname{int} V_{1}$ for some $N \in \mathbf{N}$, then $D_{N} \cap \partial V_{1} \neq \emptyset$ and since $D_{N} \cap B=\emptyset$, necessarily $D_{N}$ intersects the other boundary component, say $\Gamma$, of $V_{1}$. Then we have $\overline{D_{N}} \cap \Gamma=D_{N} \cap \Gamma$, and hence $\Gamma \subset D_{N}$ by connectedness of $\Gamma$. It follows that $D_{n} \cap \Gamma=\emptyset$ and therefore $D_{n} \subset \operatorname{int} V_{1}$ for $n>N$. Consequently,

$$
A_{2} \subset \overline{\psi\left(\mathbf{R} \times\left[s_{N+1},+\infty\right)\right)}=\overline{\bigcup_{n>N} D_{n}} \subset V_{1} \subset V
$$

for every closed annular tubular neighbourhood $V$ of $B$. This proves that $A_{2}=$ $C(x) \cup\left\{e^{+}(C)\right\}$ and similarly one can prove that if $y \in A_{1} \cap M$ then $A_{1}=$ $C(y) \cup\left\{e^{+}(C)\right\}$. Since $\partial A \subset\left(A_{1} \cap M\right) \cup\left(A_{2} \cap M\right)$, we conclude that $\partial A$ consists of at most two orbits.

If now $x \in A_{2} \cap M$ as above, then $x \in \partial C$ and one can use the same arguments as in the proof of Lemma 3.9 to prove that there is an annular tubular neighbourhood $V$ of $B$ such that $V \cap C=V \cap \bar{A}$ (closure in $M$ ). Similarly for $y \in A_{1} \cap M$. This shows that $\bar{A}$ is open and closed in $C$, hence $C=\bar{A}$. The rest of the proposition is obvious.

We are now in a position to describe topologically the closure $C \cup\left\{e^{+}(C)\right\}$ of a connected component $C$ of $R$ in $M^{+}$. If int $C=\emptyset$, then $C \cup\left\{e^{+}(C)\right\}$ is a simple closed curve. We denote by $\mathcal{R}_{0}$ the class of such connected components. If $\operatorname{int} C \neq \emptyset$ and $\partial C$ is connected, then $C \cup\left\{e^{+}(C)\right\}$ is homeomorphic to $[0,1] \times[0,1]$ with $[0,1] \times\{1\} \cup\{0,1\} \times[0,1]$ identified to a point. Thus, it is a closed disc in $M^{+}$, as shown in figure 2(a). The class of these connected components of $R$ will be denoted by $\mathcal{R}_{1}$. If $\operatorname{int} C \neq \emptyset$ and $\partial C$ has two connected components, then $C \cup\left\{e^{+}(C)\right\}$ is homeomorphic to $[0,1] \times[0,1]$ with $\{0,1\} \times[0,1]$ identified to a point. So it has the homotopy type of a simple closed curve and looks like figure 2(b). We denote the class of these connected components of $R$ by $\mathcal{R}_{2}$.

Corollary 3.12. If $C$ is a connected component of $R$, then its closure in $M^{+}$has a neighbourhood basis consisting of invariant connected open sets $V_{n}, n \in \mathbf{N}$, such that $\partial V_{n}$ consists of one or two periodic orbits for every $n \in \mathbf{N}$.

Proof. The closure of $C$ in $M^{+}$is $C \cup\left\{e^{+}(C)\right\}$, by Lemma 3.3. If $\operatorname{int} C=\emptyset$, it is a simple closed curve, by Corollary 3.10, and the proof shows that it has a neighbourhood basis consisting of annuli bounded from periodic orbits. If $\operatorname{int} C \neq$ $\emptyset$, then $\partial C$ consists of at most two orbits by Proposition 3.11. If $x \in \partial C$, there are periodic points $x_{n} \rightarrow x$ and closed annuli $W_{n}, n \in \mathbf{N}$, in $M^{+}$bounded by $C(x)$ and 


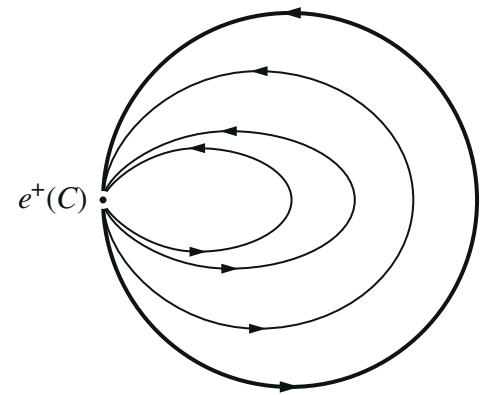

(a)

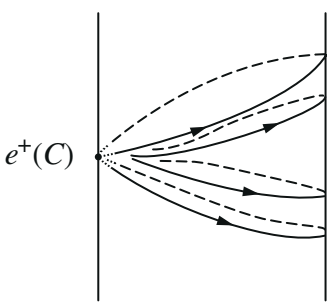

(b)

Figure 2.

$C\left(x_{n}\right)$ respectively, such that $W_{n} \cap C=C(x)$, by Lemma 3.9. If $\partial C=C(x)$, that is $\partial C$ has only one connected component, then $V_{n}=\operatorname{int} W_{n} \cup C$ is an invariant open connected neighbourhood of $C \cup\left\{e^{+}(C)\right\}$ and $\partial V_{n}=C\left(x_{n}\right)$. If $\partial C$ has another connected component $C(y)$, we have periodic points $y_{n} \rightarrow y$ and closed annuli $U_{n}$, $n \in \mathbf{N}$, bounded by $C(y)$ and $C\left(y_{n}\right)$ respectively, such that $U_{n} \cap C=C(y)$. Then we set $V_{n}=\operatorname{int} W_{n} \cup \operatorname{int} U_{n} \cup C$ and thus $\partial V_{n}=C\left(x_{n}\right) \cup C\left(y_{n}\right)$. It is clear that in both cases $\left\{V_{n}: n \in \mathbf{N}\right\}$ is a neighbourhood basis of $C \cup\left\{e^{+}(C)\right\}$ in $M^{+}$.

Corollary 3.13. If $C_{1}$ and $C_{2}$ are two disjoint connected components of $R$, then $e^{+}\left(C_{1}\right) \neq e^{+}\left(C_{2}\right)$.

Proof. The closure of $C_{1}$ in $M^{+}$has a neighbourhood basis $\left\{V_{n}: n \in \mathbf{N}\right\}$ in $M^{+}$ consisting of invariant open sets such that $\partial V_{n}$ consists of one or two periodic orbits for every $n \in \mathbf{N}$. Since $C_{1} \neq C_{2}$, if $e^{+}\left(C_{1}\right)=e^{+}\left(C_{2}\right)$ and $x \in C_{2}$, there is some $n \in \mathbf{N}$ such that $x \notin V_{n}$. But then we must have $\emptyset \neq C(x) \cap \partial V_{n} \subset P$, by connectedness of $C(x)$, because $\lim _{t \rightarrow+\infty} t x=e^{+}\left(C_{2}\right)$. This contradiction proves the corollary.

Corollary 3.14. The set $\bigcup_{C \in \mathcal{R}_{1}} C$ is closed in $M$.

Proof. Let $x \in M, C_{n} \in \mathcal{R}_{1}$ and $x_{n} \in C_{n}, n \in \mathbf{N}$, be such that $x_{n} \rightarrow x$. Then $x$ belongs to some connected component $C$ of $R$. It suffices to prove that eventually $C_{n}=C$. Suppose the contrary. Then $x \in \partial C$ and we may assume that $C_{n} \neq C$ for every $n \in \mathbf{N}$. Let $\left\{V_{n}: n \in \mathbf{N}\right\}$ be the neighbourhood basis of $C \cup\left\{e^{+}(C)\right\}$ in $M^{+}$given by Corollary 3.12. Then, $\partial V_{n}$ consists of one or two periodic orbits and at least one of them bounds with $C \cup\left\{e^{+}(C)\right\}$ an annulus $W_{n} \subset M^{+} \backslash C$. Fixing $n \in \mathbf{N}$, connectedness implies that there is some $k_{0} \in \mathbf{N}$ such that $C_{k} \cup\left\{e^{+}\left(C_{k}\right)\right\} \subset W_{n}$ for $k \geq k_{0}$. Applying again Corollary 3.12 for 
each $C_{k}$, we get a sequence of periodic orbits $C\left(y_{k}\right), k \geq k_{0}$, which bound closed discs $D_{k}$ in $W_{n}$, because $C_{k} \in \mathcal{R}_{1}$, and $x_{k} \in D_{k}$ for every $k \geq k_{0}$. It follows that $y_{k} \rightarrow x$ and we arrive at a contradiction as in case I of the proof of Lemma 3.9.

Note that $R$ may have uncountably many connected components. Consider, for example, the planar ordinary differential equation (in polar coordinates)

$$
\dot{r}=0 \text { and } \dot{\theta}=f(r, \theta)
$$

where $f: \mathbf{R}^{2} \rightarrow[0,1]$ is a smooth function such that $f^{-1}(0)$ is the standard Cantor set $X$ on $\theta=0$. The restricted flow on $M=\mathbf{R}^{2} \backslash X$ is $D$-stable and $R$ has uncountably many connected components with empty interiors. Note also that an orbit in the boundary of $R$ may not be in the boundary of any connected component of $P$.

\section{The orientable 2-manifolds of finite genus admiting $D$-stable flows}

In this section we shall use the analysis of the dynamics of the preceeding section 3 to determine topologically the orientable 2 -manifolds of finite genus that admit non-minimal $D$-stable flows. For the rest of the section we assume that $M$ is an orientable 2-manifold of finite genus and we continue to use the notation of section 3 for a given $D$-stable flow.

Note that if $M$ is compact and admits a non-minimal $D$-stable flow $\phi$, then $J^{+}(x)=L^{+}(x)$ for every $x \in M$, that is the flow is $D^{+}$-stable in the sense of [2], and therefore $M$ must be homeomorphic to the 2-sphere $S^{2}$ or the 2-torus $T^{2}$, by [2, Corollary 3.4]. On the other hand, if $M$ admits a $D$-stable flow with $R=M$, then $M$ is homeomorphic to $\mathbf{R}^{2}$ or to $\mathbf{R} \times S^{1}$, by Theorem 3.4. Thus, we proceed making the assumption that $M$ is non-compact and not homeomorphic to $\mathbf{R}^{2}$ or $\mathbf{R} \times S^{1}$.

Lemma 4.1. If $M$ admits a non-minimal D-stable flow, then it admits a nonminimal D-stable flow with the same number of singularities such that the set of points with non-compact orbits has no connected component in class $\mathcal{R}_{1}$.

Proof. Let $\phi$ be a non-minimal $D$-stable flow on $M$. From Corollary 3.14 , the set

$$
N=M \backslash \bigcup_{C \in \mathcal{R}_{1}} C
$$

is an open invariant subset of $M$. Moreover $N$ is homeomorphic to $M$ and the restricted flow on it is $D$-stable. By the definition of $N$, no connected component of the set of points in $N$ with non-compact orbits is in class $\mathcal{R}_{1}$. 
Proposition 4.2. If $M$ admits a non-minimal D-stable flow, then it admits a non-minimal D-stable flow with the same number of singularities, and whose set of points with non-compact orbits has empty interior.

Proof. Let $\phi$ be a non-minimal $D$-stable flow on $M$. Our assumption that $M$ is non-compact and not homeomorphic to $\mathbf{R}^{2}$ or $\mathbf{R} \times S^{1}$ implies that $\phi$ has periodic orbits. Let $R_{\phi}$ denote the set of points of $M$ whose orbit with respect to $\phi$ is non-compact. Let $S$ be a section to the restricted flow in $R_{\phi}$, which exists because of Proposition 3.5. As we showed in section 3 and by Lemma 4.1, we may assume that the connected components of $R_{\phi}$ with non-empty interior belong to class $\mathcal{R}_{2}$. If $C \in \mathcal{R}_{2}$, the restricted flow in $C$ is parallelizable with section $S_{C}=S \cap C$, which is homeomorphic to the interval $[0,1]$. Corollary 3.13 implies that the family

$$
\mathcal{D}=\left\{\{x\}: x \in M^{+} \backslash \bigcup_{C \in \mathcal{R}_{2}} C\right\} \cup\left\{t S_{C}: t \in \mathbf{R}, C \in \mathcal{R}_{2}\right\} \cup\left\{\left\{e^{+}(C)\right\}: C \in \mathcal{R}_{2}\right\}
$$

is a decomposition of $M^{+}$into compact contractible subsets. We shall show that $\mathcal{D}$ is upper semicontinuous, meaning that if $A \in \mathcal{D}$ and $W \subset M^{+}$is an open set containing $A$, there is an open subset $V$ of $M^{+}$which a union of elements of $\mathcal{D}$ such that $A \subset V \subset W$.

Suppose by contradiction that this is not true for some $A \in \mathcal{D}$ and some open neighbourhood $W$ of $A$ in $M^{+}$with $\partial W \subset M$. By compactness of $A$ there are a point $x \in A$ and $x_{n} \rightarrow x$ such that $\mathcal{D}\left(x_{n}\right) \not \subset W$. Since $x_{n} \in W$, eventually, this means that we may assume that $\mathcal{D}\left(x_{n}\right)=t_{n} S_{C_{n}}$ for some $t_{n} \in \mathbf{R}$ and $C_{n} \in \mathcal{R}_{2}$, from the definition of $\mathcal{D}$. By connectedness of $S_{C_{n}}$, there are $y_{n} \in S_{C_{n}}$ such that $t_{n} y_{n} \in \partial W$ for every $n \in \mathbf{N}$. Since $\partial W$ is compact, passing to a subsequence if necessary, we may assume that there is a point $z \in R \cap \partial W$ such that $t_{n} y_{n} \rightarrow z$. It follows that there are $t \in \mathbf{R}$ and $y \in R$ such that $t_{n} \rightarrow t$ and $y_{n} \rightarrow y$, because the restricted flow in $R$ is parallelizable. Hence $z=t y$. Let $C$ be the connected component of $R$ that contains $z$. We have to deal with the following two cases.

Case I. If $x \in M$, then $x \in t S$ and $e^{+}(x)=e^{+}(z)$, by Proposition 2.3. Hence $x \in t S_{C}$, from Corollary 3.13. If $C \in \mathcal{R}_{0}$, then $C=C(x)=C(z)$, from Corollary $3.10, x \neq z$ and $\{x, z\} \subset C(x) \cap t S$, contradiction. If $C \in \mathcal{R}_{2}$, then $A=\mathcal{D}(x)=$ $t S_{C}$. But then $z \in A \cap \partial W=\emptyset$, contradiction again.

Case II. If $x \in M^{+} \backslash M$, then $\mathcal{D}(x)=\{x\}$. Moreover, we may assume that $C_{n} \neq C$ for every $n \in \mathbf{N}$. It follows that $z, y \in \partial C$. Let $U$ be a compact neighbourhood of $y$ in $M$ which does not intersect the other boundary component of $C$, if any. We have eventually $S_{C_{n}} \cap \partial U \neq \emptyset$, because $x_{n} \rightarrow x$ and $y_{n} \rightarrow t \in \mathbf{R}$. If $z_{n} \in S_{C_{n}} \cap \partial U$, the sequence $\left\{z_{n}: n \in \mathbf{N}\right\}$ has a limit point $z_{0} \in S \cap \partial U$, and $e^{+}\left(z_{0}\right)=e^{+}(y)$, from Proposition 2.3. Therefore, $z_{0} \in \partial C \cap \partial U$, by Corollary 3.13. Our choice of $U$ implies that $z_{0} \in C(y) \cap S=\{y\}$. This contradiction proves the upper semicontinuity of $\mathcal{D}$.

According now to [11, Theorem 1], the quotient space $M^{+} / \mathcal{D}$ is homeomorphic to $M^{+}$. Intuitively, $M^{+} / \mathcal{D}$ is obtained by shrinking each $C \in \mathcal{R}_{2}$ to an orbit, 
along a section. Note that the extended flow on $M^{+}$preserves $\mathcal{D}$ and so induces a flow on $M^{+} / \mathcal{D}$, which makes the identification map $p: M^{+} \rightarrow M^{+} / \mathcal{D}$ an epimorphism of dynamical systems. It is obvious from the definition of $\mathcal{D}$ that $p$ maps $M^{+} \backslash M$ in a one-to-one manner onto $p\left(M^{+} \backslash M\right)$. Thus, the invariant set $N=M^{+} / \mathcal{D} \backslash p\left(M^{+} \backslash M\right)$ is homeomorphic to $M$. However, $N=p(M)$ from the definition of $\mathcal{D}$, and therefore $p \mid M: M \rightarrow N$ is a homomorphism of $\phi$ onto the restricted flow in $N$, which we denote by $\psi$. Note that $\{\mathcal{D}(x): x \in M\}$ is an upper semicontinuous decomposition of $M$ into compact sets. This is equivalent to saying that $p \mid M$ is a proper map. Thus, $\psi$ is a $D$-stable flow by Proposition 2.2. It remains to prove that int $R_{\psi}=\emptyset$, where $R_{\psi}$ is the set of non-compact orbits of $\psi$. If $\operatorname{int} R_{\psi} \neq \emptyset$, then $p^{-1}$ (int $R_{\psi}$ ) is a non-empty, open subset of $R_{\phi}$, and is a union of elements of $\mathcal{D}$. If $x \in p^{-1}\left(\operatorname{int} R_{\psi}\right)$, then $p^{-1}\left(\operatorname{int} R_{\psi}\right)$ is an open neighbourhood of $\mathcal{D}(x)$ containd in $R_{\phi}$. But Corollary 3.12 and the definition of $\mathcal{D}$ imply that there is no such neighbourhood.

Theorem 4.3. An orientable 2-manifold of finite genus $M$ admits a non-minimal $D$-stable flow if and only if $M$ has genus 0 or 1.

Proof. If $M$ is compact, then it is homeomorphic to $S^{2}$ or $T^{2}$. If it is non-compact and admits a parallelizable $D$-stable flow, then it is homeomorphic to $\mathbf{R}^{2}$ or $\mathbf{R} \times S^{1}$. So in these cases $M$ has genus 0 or 1 . If $M$ is non-compact and not homeomorphic to $\mathbf{R}^{2}$ or $\mathbf{R} \times S^{1}$ and admits a non-minimal $D$-stable flow, then, by Proposition 4.2 , it admits a non-minimal $D$-stable flow with the same number of singularities and whose set $R$ of points with non-compact orbits has $\operatorname{int} R=\emptyset$. Let $\tilde{M}=$ $M \cup\left\{e^{+}(x): x \in R\right\}$. We shall show that $\tilde{M}$ is open in $M^{+}$.

Since $\operatorname{int} R=\emptyset$, every connected component $C$ of $R$ is a single orbit and $C \cup$ $\left\{e^{+}(C)\right\}$ is a simple closed curve which has a neighbourhood basis in $M^{+}$consisting of invariant open annuli bounded by periodic orbits. Let $V$ be such an annulus. It suffices to prove that $V \cap\left(M^{+} \backslash M\right) \subset \tilde{M}$. Let $e \in V \cap\left(M^{+} \backslash M\right)$ and $D$ be a closed disc neighbourhood of $e$ such that $D \subset V$ and $\partial D \subset M$. As in case I of the proof of Lemma 3.9, there is no periodic orbit in $V$ which is nullhomotopic in $V$. It follows from this and the assertion of Lemma 3.9 that $D$ contains no complete orbit in $M$. According to [4, Ch. VI, Theorem 1.1], there exist ends $e_{n} \in D$ and points $x_{n} \in \partial D$ such that $e_{n} \rightarrow e$ and $e^{+}\left(x_{n}\right)=e_{n}$, for every $n \in \mathbf{N}$. Since $\partial D$ is compact, we may assume that there is a point $x \in \partial D$ such that $x_{n} \rightarrow x$. Then, $x \in R$ and there are $t_{n} \rightarrow+\infty$ such that $t_{n} x_{n} \rightarrow e$. From Corollary 3.6 we have $e=e^{+}(x)$. This shows that $\tilde{M}$ is an open subset of $M^{+}$, and it is obvious that it is invariant under the extended flow on $M^{+}$. Note also that $\tilde{M}$ and $M$ have the same genus.

The family of disjoint simple closed curves 


$$
\mathcal{F}=\{C(x): x \in P\} \cup\left\{C(x) \cup\left\{e^{+}(x)\right\}: x \in R\right\}
$$

fills $\tilde{M} \backslash F$, where $F$ is the set of singularities and $P$ the set of periodic points of the flow in $M$. It follows from Corollary 3.12 , that $\mathcal{F}$ is an oriented regular family of curves in the sense of $\mathrm{H}$. Whitney, and so there is a flow on $\tilde{M}$ whose orbits in $\tilde{M} \backslash F$ are the elements of $\mathcal{F}$ and $F$ is the set of its singularities [12]. From Corollary 3.12 follows immediately that this flow on $\tilde{M}$ is $D^{+}$-stable in the sense of [2]. Since each orbit is singular or periodic, it follows that if $F \neq \emptyset$, then $\tilde{M}$ is homeomorphic to $S^{2}$ or $\mathbf{R}^{2}$ and $F$ consists of one or two centers, by [2, Theorem 3.1.4], and if $F=\emptyset$, then $\tilde{M}$ is homeomorphic to $\mathbf{R} \times S^{1}$ or $T^{2}$, by [2, Theorem 3.1.5]. In every case $\tilde{M}$ has genus 0 or 1 , and so has $M$.

From the proof of Theorem 4.3 we get the following information about the number of singularities.

Theorem 4.4. A D-stable flow on an orientable 2-manifold of finite genus can have at most two singularities and they are necessarily centers. Moreover, the existence of a singularity implies that the underlying manifold has genus 0 .

It is difficult to state a theorem describing the global qualitative behavior of a $D$-stable flow on an orientable 2-manifold of finite genus and covering all possible cases. However, the cases occuring if the underlying manifold is compact are easily described as in [2] and the results of section 3 with Theorems 4.3 and 4.4 give a picture of what the typical phase portrait of a non-minimal $D$-stable flow on a non-compact 2-manifold of finite genus looks like.

\section{References}

[1] H. Abels, Enden von Räumen mit eigentlichen Transformationsgruppen, Comment. Math. Helvetici. 47 (1972), 457-473.

[2] K. Athanassopoulos, $D^{+}$-stable dynamical systems on 2-manifolds, Math. Z. 196 (1987), 453-462.

[3] K. Athanassopoulos and A. Manoussos, Minimal flows on multipunctured surfaces of infinite type, Bull. London Math. Soc. 27 (1995), 595-598.

[4] N.P. Bhatia and G.P. Szegö, Stability theory of dynamical systems, Springer-Verlag, New York 1970.

[5] C. Gutierrez, Smoothing continuous flows on two manifolds and recurrences, Ergodic Th. Dynamical Systems 6 (1986), 17-44.

[6] O. Hajek, Dynamical systems in the plane, Academic Press, New York 1968.

[7] R. Knight, Prolongationally stable transformation groups, Math. Z. 161 (1978), 181-194.

[8] R.J. Knill, A $C^{\infty}$ flow on $S^{3}$ with a Denjoy minimal set, J. Diff. Geom. 16 (1981), 271-280.

[9] E. Lima, Common singularities of commuting vector fields on 2-manifolds, Comment. Math. Helvetici. 39 (1964), 97-110.

[10] Th. Petrescou, Contribution to the theory of $D$-stable dynamical systems on 2-manifolds, Thesis, Univ. of Athens, 1989 (in Greek). 
[11] J.H. Roberts and N.E. Steenrod, Monotone transformations of two-dimensional manifolds, Ann. of Math. 39 (1938), 851-862.

[12] H. Whitney, Regular families of curves, Ann. of Math. 34 (1933), 244-270.

[13] T.-S. Wu, Continuous flows with closed orbits, Duke Math. J. 31 (1964), 463-469.

Konstantin Athanassopoulos Department of Mathematics University of Crete GR-71409 Iraklion, Crete Greece

Theodoros Petrescou Naval Military Academy GR-18539 Piraeus

Greece

(Received: January 13, 1997)
Polychronis Strantzalos Department of Mathematics University of Athens GR-15784 Panepistimiopolis Greece 